\title{
Differential Diagnosis and Treatment of Septic Arthritis in the Temporomandibular Joint: A Case Report and Literature Review
}

\author{
Bola Kim, Hyo-Won Choi, Jae-Young Kim, Kwang-Ho Park, Jong-Ki Huh
}

Department of Oral and Maxillofacial Surgery, Gangnam Severance Hospital, Yonsei University College of Dentistry, Seoul, Korea

Received August 8, 2019

Revised August 30, 2019

Accepted August 30, 2019
Septic arthritis of the temporomandibular joint (TMJ) is an uncommon disease caused by microbial pathogens through hematogenous infection, local spread, or iatrogenic infection. As the symptoms have an insidious onset, the early stage of septic arthritis is often confused with other diseases. A 49-year-old man was referred for increasing preauricular pain, swelling, and restricted mouth opening. He had been initially diagnosed as having a conventional temporomandibular joint disorder and trigeminal neuralgia and had been treated for the same. Imaging studies including panoramic view, lateral tomography, computed tomography with contrast enhancement, and magnetic resonance imaging were performed. Erosive bone change with displacement of the involved condyle, diffuse swelling of adjacent soft tissue, and fluid collection in the joint space were noted. Needle aspiration of the joint space and bacterial culture confirmed the diagnosis of septic arthritis of the TMJ and he was treated with antibiotic therapy and surgical drainage. Clinicians should always consider the diagnosis of septic arthritis of the TMJ in patients with preauricular pain or swelling.

Key Words: Arthritis; Arthrocentesis; Infectious; Temporomandibular joint; Temporomandibular joint disorders

\section{INTRODUCTION}

Septic arthritis is a rare disease caused by a microbial pathogen in the joint space. It usually affects the large joints, and infection of the temporomandibular joint (TMJ) has been very rarely reported [1-4]. It is characterized by an insidious onset and a gradual infective process. The microbial pathogens can spread by the hematogenous route in patients with systemic infection, from local infections, and through iatrogenic inoculation $[5,6]$.

The common clinical signs of septic arthritis in the TMJ are localized heat, edema, erythema, and pain in the preauricular area, which are similar to the signs of local inflammation [7]. The symptoms also include ipsilateral posterior open bite, contralateral deviation of the mandible, and limitation of joint movements, which can be confused with conventional temporomandibular disorder (TMD). Increased incidence of complications, such as destruction of the involved joint structure has been reported when adequate treatment is delayed [7,8]. Early differential diagnosis and proper management of the condition is required.

Generally, the differential diagnosis of septic arthritis should include common TMDs, cellulitis of the adjacent soft tissues, malignant otitis externa, gout, pseudogout, rheumatologic disease, traumatic effusions, neoplasms, such as ganglion cysts, parotid tumors, and synovial chondromatosis [1,9-11].

Here we present a patient with septic arthritis of the TMJ, which was misdiagnosed as conventional TMD and trigeminal neuralgia, with literature review. 


\section{CASE REPORT}

A 49-year-old man was referred from the Department of Otorhinolaryngology for further evaluation of a swelling over the left preauricular area. He had poorly controlled diabetes. The patient had developed a pain on the left side of his face and teeth 2 months prior to the consultation, which worsened on mouth opening. He visited a dental clinic and was diagnosed to have TMD for which he received conservative management. As the symptoms did not improve, he visited the Department of Pain Medicine, where he was diagnosed to have trigeminal neuralgia and received nerve block treatment. The pain initially decreased for a few days, but 2 weeks ago, the pain worsened again and there was malocclusion of the left molars. Three days ago, he experienced difficulty in opening the mouth and developed a swelling on his left cheek.

On clinical examination, there was diffuse swelling, induration, localized warmth, tenderness, and redness on the left side of his face. The range of maximum mouth opening was $16 \mathrm{~mm}$, with worsening of the pain on mouth opening. There was open bite on the ipsilateral molar area. Dental caries was detected in the right maxillary molars, but the teeth on the left maxilla and mandible showed no percussion and mobility signs. No other specific sign was noted in the intraoral area (Fig. 1).

Vitals signs were in the normal range except for a slight fever $\left(37^{\circ} \mathrm{C}\right)$ at first. Hematological laboratory tests were performed for testing severity of the infection. The results showed inflammatory signs, including elevation of white blood cell counts, 18,920/ $\mu \mathrm{L}$; neutrophil ratio, 76.4\%; erythrocyte sedimentation rate (ESR), $63 \mathrm{~mm} / \mathrm{h}$; and serum C-reactive protein, $61.8 \mathrm{mg} / \mathrm{L}$ (the normal ranges of the laboratory tests are as follows: white blood cell counts, 4,00010,800/ $\mu \mathrm{L}$; neutrophil ratio, 40\%-73\%; ESR, 0-15 mm/h; and serum C-reactive protein, 0.1-6.0 mg/L).

Imaging studies including panoramic view, lateral tomography, computed tomography (CT) with contrast enhancement, and magnetic resonance imaging (MRI) were performed. On the panoramic view and lateral tomography images, the left condyle was slightly displaced in the anterior and downward direction. The continuity of the cortical bone was broken at the posterior condylar neck. The CT image showed diffuse swelling and fluid collection in the soft tissue over the left TMJ and adjacent masticatory space, and on the MRI image, a low signal area which was thought to be air bubbles or fluid collection was noted adjacent to the condylar head. Additionally, the high signal area of adjacent soft tissue showed increased vascularity owing to vasodilation or edematous change (Fig. 2-4).

To confirm the diagnosis, needle aspiration of the left joint space was performed with a $5 \mathrm{~mL}$ syringe under local anesthesia, and about $1.5 \mathrm{~mL}$ of yellowish-brown fluid was aspirated (Fig. 5). Septic arthritis of the left TMJ was confirmed and the aspirated fluid was sent to the laboratory for culture and antibiotic susceptibility tests. Intravenous antibiotic therapy under admission was planned. Cefotetan, which is a second-generation, was used intravenously as

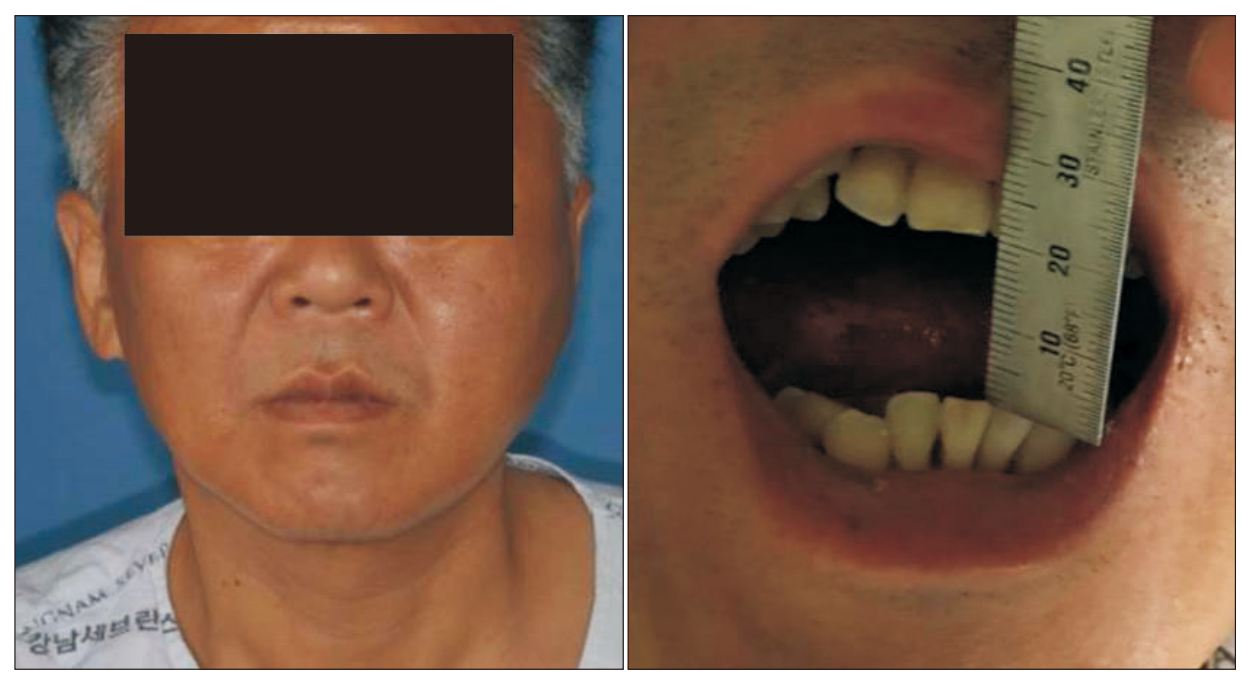

Fig. 1. Swelling of the left preauricular area with restricted jaw movement. 
the first choice before the culture and sensitivity reports were available. Culture of the abscess fluid showed growth of Haemophilus aphrophilus. Considering the antibiotic sensitivity report, the antibiotic was changed to ambactam, which includes ampicillin and sulbactam.

Surgical intervention comprising drainage and debridement

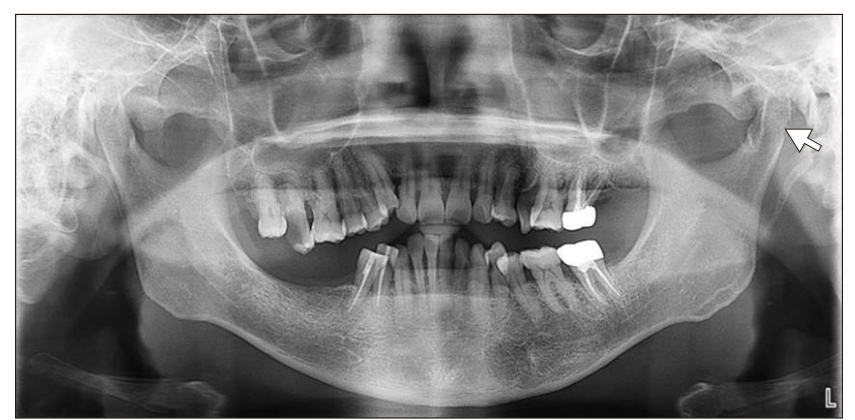

Fig. 2. Panoramic view showing joint space widening of the left temporomandibular joint and the discontinuity of cortical bone of the left condyle (arrow). was planned. Under antibiotic coverage and local anesthesia, the incision and drainage procedures and insertion of a surgical drain were performed via the extraoral approach. The symptoms showed a little improvement, but did not resolve completely; thus, open surgery was planned for examination of the inner structures, drainage of the inflammatory exudate, and debridement of the joint space. Under general anesthesia, a $5 \mathrm{~cm}$ incision and blunt dissection was performed via the preauricular approach. The capsular structure of the TMJ was found to be partially destroyed with inflammatory tissue and purulent abscess formation. The disc structure of the TMJ was perforated and softened in the center with degenerative rough margins on the condylar head. High volume irrigation as well as debridement of the softened disc structure and inflammatory tissue was performed. Abscess formed at the adjacent masticatory space was also completely irrigated.

The surgical drain was removed and the patient was
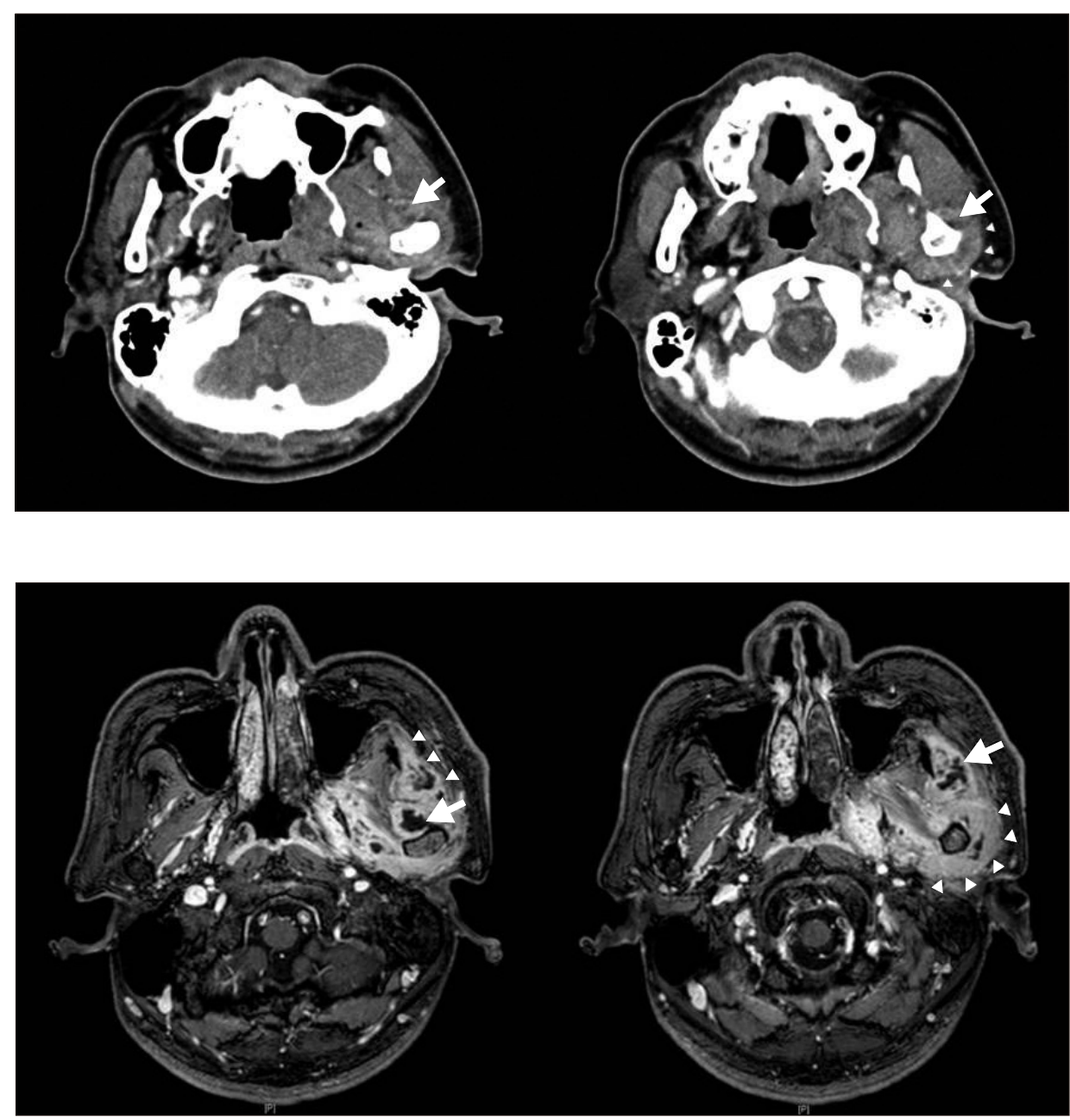

Fig. 3. Computed tomography shows fluid collection (arrow) and diffuse swelling of adjacent soft tissue (arrowhead).
Fig. 4. T1-weighted magnetic resonance images show a low signal area which is thought to be an air bubble or fluid collection (arrow) and a high signal area which is thought to be edematous change in the adjacent soft tissue (arrowhead). 


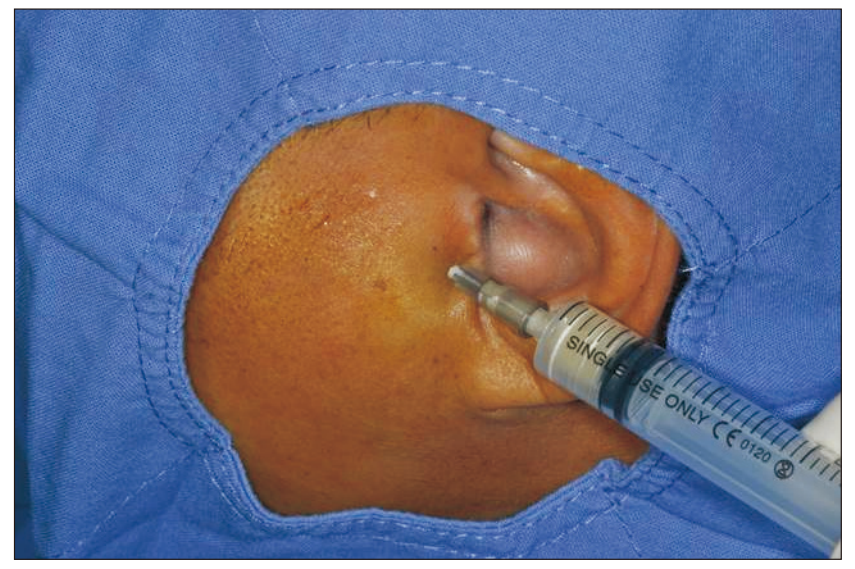

Fig. 5. Yellowish fluid aspirated from the joint space.

discharged after the C-reactive protein levels and white blood cell counts were normalized and his symptoms had improved. Following discharge, the patient was instructed on regular physical therapy including active mouth opening, and periodic follow-up was performed every 2 weeks until there was recovery in the range of mouth opening. At 2 months follow-up, the range of maximum mouth opening was $47 \mathrm{~mm}$.

\section{DISCUSSION}

Septic arthritis of the TMJ is an infection of the TMJ caused by a microbial pathogen, which spreads to the joint space from adjacent infectious tissues or hematogenously spreads following pharyngitis, upper respiratory infection, or dental infections [7,9]. In rare cases, iatrogenic infection caused by local inoculation has been reported $[5,6]$. In this case, the patient had an insidious onset of the infection with mild symptoms over a long period and the diagnosis was delayed. The exact route of spread of infection was, therefore, not clear. Considering patient's medical history of nerve block treatment, the possibility of iatrogenic infection is low but cannot be excluded. There are local as well as general predisposing factors for septic arthritis of the TMJ. The local factors include blunt trauma, burn wounds, and iatrogenic causes. The general factors are autoimmune disease (rheumatoid arthritis, diabetes, immunosuppression, and hypogammaglobulinemia), prolonged use of systemic steroids, and sexually transmitted diseases.

The manifestations of septic arthritis of the TMJ include local heat, edema, erythema, pain over the preauricular area, limitation of jaw movement, malocclusion with ipsilateral posterior open bite, and regional lymphadenopathy [7]. However, the disease usually has an insidious onset and only a few of these symptoms are manifested in the early stage. There are several case reports about patients with septic arthritis in whom the diagnosis was confused with common TMDs and other diseases. Gayle et al. [4] reported a patient who was misdiagnosed as having a common TMD and for whom proper management was delayed. Gams and Freeman [12] also reported a patient with septic arthritis previously treated with muscle relaxants and analgesics for trismus. In the present case, the symptom of pain over the preauricular area worsening at mouth opening was confused with that of conventional TMD. Also, the continuous severe pain was misdiagnosed as trigeminal neuralgia. However, the patient had no previous TMD history and his symptoms developed suddenly and worsened gradually. Moreover, the nerve block treatment for trigeminal neuralgia initially reduced his symptoms, but they soon worsened. If the treating clinicians had considered septic arthritis of the TMJ in the differential diagnosis, it would have been possible to diagnose the condition early with further evaluation.

To diagnose septic arthritis of the TMJ, signs of infection should be examined for differentiating from common TMDs or neoplasms through laboratory tests. The key point for diagnosing septic arthritis of the TMJ is needle aspiration of the joint space for detection of any abscess formed by the microbial organism. Early diagnosis performed by needle aspiration examination is required for early treatment and antibiotic selection. According to the literature, recommendations for diagnosis of septic arthritis are as follows. First of all, history taking and physical examination is mandatory. Joint fluid aspiration with culture (aerobic and anaerobic) and gram stain examination as well as blood culture are also required. Laboratory tests including white blood cell counts, ESR, and C-reactive protein, radiographs (plain film, CT), bone scan, and MRI can help with the diagnosis. Also, it is important to consider the response to treatment $[1,7]$.

Imaging study is required for examination of the involved structures as well as determining the severity. Panoramic 
images show joint space widening due to increased synovial fluid, inflammatory exudate, and abscess formation. Therefore, displacement of the condylar head either anteriorly or inferiorly should be assessed [13,14]. In severe infections, destruction of the involved bony structures and disc structure can be detected by imaging studies. CT provides more accurate images of abscess formation in the joint space, involved tissue changes, and bone destruction than radiography. It should be the imaging modality of first choice for diagnosing septic arthritis of the TMJ. MRI can provide information about the exudate, abscess formation in the joint, and especially the extent of bony involvement initially. In this study, initial imaging studies with CT and MRI were performed and destruction of the posterior cortical bone of the condyle caused by delayed diagnosis and treatment was noted7.

Systemic administration of antibiotics is required to control the infection above all [15]. Penicillin with a $\beta$-lactamase inhibitor is recommended for initial empirical therapy for septic arthritis of the TMJ, which can then be modified based on the culture and sensitivity results as well as the patient's clinical response. Long-term antibiotic therapy for 1 month is recommended [1,7]. The causative organisms for septic arthritis of the TMJ include Staphylococcus aureus, Neisseria gonorrhoeae, Staphylococcus, Streptococcus, and Haemophilus influenzae $[1,14]$. The gram stain test and microbial sensitivity tests should be performed. In this case, Haemophilus aphrophilus was found in the abscess fluid. Haemophilus influenzae is a gram-negative facultative coccobacillus found in the oral cavity with low pathogenicity [16,17]. Merino et al. [17] have reported that dental diseases can cause of transient bacteremia and distant Haemophilus influenzae infection. Huang et al. [16] have reported Haemophilus influenzae to be susceptible to antibiotics, such as ceftriaxone, cefotaxime, and fluoroquinolones.

Surgical intervention is required for drainage of the inflammatory exudate and abscess, decompression of the joint space, and direct application of antibiotics. The decision depends on the severity of the symptoms in the patient. Needle aspiration, arthrocentesis, arthroscopy, and open surgery are the options of surgical intervention depending on the situation. Needle aspiration is used for initially determining the antimicrobial of choice and for providing some decompression without a complicated operation. Goldenberg et al. [18] compared needle aspiration with surgical drainage in the treatment of septic arthritis in other joints and there was no statistically significant difference in the results. Arthrocentesis and arthroscopy have been reported to have advantages of efficiency and low morbidity in many studies $[3,19]$. In this case, several stages of surgical intervention were planned, starting from needle aspiration and arthrocentesis under local anesthesia to open surgery. Clinicians should consider the range and severity of the infection. Also, the patient's response to treatment should be considered to determine which kind of surgical intervention is needed [18].

In patients with septic arthritis of the TMJ, an early diagnosis should be emphasized because delayed diagnosis and treatment can result in irreversible complications, including TMJ dysfunction, fibrosis, ankylosis, infection spread, and mandibular growth alteration [7]. Ward et al. [20] reported that the treatment results were poorer in patients in whom the treatment had been delayed by 7 or more days compared to that in patients who had early treatment. This is because inflammatory exudate and abscess increase the joint compression resulting in necrosis of the articular structures. In addition, bacterial penetration into the joint results in an inflammatory cytokine response, which causes irreversible destruction within 7 days $[1,8,18]$. In this case, the proper diagnosis and treatment were delayed, resulting in involved condylar destruction as shown by the imaging study and the need for long-term treatment.

Physical therapy is the indispensable sequence in the treatment of septic arthritis of the TMJ, especially for the resolution of the long-term complications. It is reported that the inflammatory status of the joint causes fibrinogen deposition, which can result in ankylosis of the TMJ when the diagnosis is delayed $[1,18]$. For recovery of the mandibular motion and remodeling of the condyle to prevent fibrosis and ankylosis, physical therapy should be performed, including anterior, lateral, and opening exercises. Early physical therapy should begin after the acute phase of the illness has passed [7]. In this case, the patient was educated about the active range-of-motion exercises and weekly assessments were performed until he recovered the 
normal range-of-motion. It took 1 month for the patient to obtain $40 \mathrm{~mm}$ maximum mouth opening and 1 month and 2 weeks for $47 \mathrm{~mm}$ mouth opening, which is considered as the normal range. It is believed that delayed treatment caused the long recovery period for his normal range-ofmotion of the mandible.

In conclusion, this was a case of septic arthritis of the TMJ in which the proper diagnosis and treatment were delayed. Clinicians should always keep in mind the possibility septic arthritis of the TMJ in patients with preauricular pain or swelling.

\section{CONFLICT OF INTEREST}

No potential conflict of interest relevant to this article was reported.

\section{ORCID}

\author{
Bola Kim \\ https://orcid.org/0000-0002-2460-8737 \\ Hyo-Won Choi \\ https://orcid.org/0000-0002-2481-889X \\ Jae-Young Kim \\ https://orcid.org/0000-0002-9423-438X \\ Kwang-Ho Park \\ https://orcid.org/0000-0003-1942-2986 \\ Jong-Ki Huh \\ https://orcid.org/0000-0002-7381-3972
}

\section{REFERENCES}

1. Goldschmidt MJ, Butterfield KJ, Goracy ES, Goldberg MH. Streptococcal infection of the temporomandibular joint of hematogenous origin: a case report and contemporary therapy. J Oral Maxillofac Surg 2002;60:1347-1353.

2. Moses JJ, Lange CR, Arredondo A. Septic arthritis of the temporomandibular joint after the removal of third molars. J Oral Maxillofac Surg 1998;56:510-512.

3. Cai XY, Yang C, Zhang ZY, Qiu WL, Chen MJ, Zhang SY. Septic arthritis of the temporomandibular joint: a retrospective review of 40 cases. J Oral Maxillofac Surg 2010;68:731-738.
4. Gayle EA, Young SM, McKenna SJ, McNaughton CD. Septic arthritis of the temporomandibular joint: case reports and review of the literature. J Emerg Med 2013;45:674-678.

5. McCain JP, Zabiegalski NA, Levine RL. Joint infection as a complication of temporomandibular joint arthroscopy: a case report. J Oral Maxillofac Surg 1993;51:1389-1392.

6. Matsumura Y, Inui M, Tagawa T. Peritemporomandibular abscess as a complication of acupuncture: a case report. J Oral Maxillofac Surg 1998;56:495-496.

7. Leighty SM, Spach DH, Myall RW, Burns JL. Septic arthritis of the temporomandibular joint: review of the literature and report of two cases in children. Int J Oral Maxillofac Surg 1993;22:292297.

8. Nade S. Acute septic arthritis in infancy and childhood. J Bone Joint Surg Br 1983;65:234-241.

9. Bounds GA, Hopkins R, Sugar A. Septic arthritis of the temporomandibular joint-a problematic diagnosis. Br J Oral Maxillofac Surg 1987;25:61-67.

10. Hekkenberg RJ, Piedade L, Mock D, Baker G, Freeman JL. Septic arthritis of the temporomandibular joint. Otolaryngol Head Neck Surg 1999;120:780-782.

11. Varghese L, Chacko R, Varghese GM, Job A. Septic arthritis of the temporomandibular joint caused by Aspergillus flavus infection as a complication of otitis externa. Ear Nose Throat $J$ 2015;94:E24-E26.

12. Gams K, Freeman P. Temporomandibular joint septic arthritis and mandibular osteomyelitis arising from an odontogenic infection: a case report and review of the literature. J Oral Maxillofac Surg 2016;74:754-763.

13. Winters SE. Staphylococcus infection of the temporomandibular joint. Oral Surg Oral Med Oral Pathol 1955;8:148-150.

14. Hincapie JW, Tobon D, Diaz-Reyes GA. Septic arthritis of the temporomandibular joint. Otolaryngol Head Neck Surg 1999;121:836-837.

15. Parker RH, Schmid FR. Antibacterial activity of synovial fluid during therapy of septic arthritis. Arthritis Rheum 1971;14:96104.

16. Huang ST, Lee HC, Lee NY, Liu KH, Ko WC. Clinical characteristics of invasive Haemophilus aphrophilus infections. J Microbiol Immunol Infect 2005;38:271-276.

17. Merino D, Saavedra J, Pujol E, et al. Haemophilus aphrophilus as a rare cause of arthritis. Clin Infect Dis 1994;19:320-322.

18. Goldenberg DL, Brandt KD, Cohen AS, Cathcart ES. Treatment of septic arthritis: comparison of needle aspiration and surgery as initial modes of joint drainage. Arthritis Rheum 1975;18:83-90.

19. Murakami K, Matsumoto K, lizuka T. Suppurative arthritis of the temporomandibular joint. Report of a case with special reference to arthroscopic observations. J Maxillofac Surg 1984;12:41-45.

20. Ward J, Cohen AS, Bauer W. The diagnosis and therapy of acute suppurative arthritis. Arthritis Rheum 1960;3:522-535. 